\title{
GPR-Jahrestagung im hohen Norden ein voller Erfolg
}

Die 56. Jahrestagung der Gesellschaft für Pädiatrische Radiologie vom 18. bis 21. September 2019 in Rostock und Warnemünde war ein voller Erfolg. Kinderradiologinnen und Radiologen, MTRA sowie Kolleginnnen und Kollegen aus benachbarten Fachdisziplinen hatten ihren Weg in den Norden gefunden und erlebten vier spannende Kongresstage.

Der Fortbildungstag für Pädiater und Radiologen am Mittwoch zum Thema „Rund um den Thorax“ sowie der Sonorefreherkurs mit Referaten, praktischen Übungen und Videodemonstrationen wurde von den Teilnehmenden sehr gut angenommen.
Das Tagungsprogramm bot ein vielfältiges Themenspektrum von „Pädiatrischer Neuroradiologie“ über „Muskuloskelettaler Bildgebung“ bis zum „Kinderschutz“. Auch für MTRA wurde ein abwechslungsreiches Programm geboten. Eine großzügige Industrieausstellung sowie zahlreiche LunchSymposien rundeten das Programm ab.

\section{Preisverleihungen}

Hanne Kirsch und Jessica Rodigas aus Jena erhielten den Publikationspreis der GPR 2019 für ihre Arbeit „Static and Functional MR Urography to Assess Congenital Anomalies of the Kidney and Urinary Tract in
Infants and Children: Comparison With MAG3 Renal Scintigraphy and Sonography“. Zwei weitere Publikationspreise wurden ebenfalls in Rostock verliehen. Dennis Dünger von der Universität Leipzig erhielt den Preis für seine Veröffentlichung „Do we need gadolinium-based contrast medium for brain magnetic resonance imaging in children?“. PD Dr. Wibke Uller erhielt den Publikationspreis 2019 der GPR für ihre Veröffentlichung „Preoperative Embolization of Venous Malformations Using n-Butyl Cyanoacrylate“.

Der Vortragspreis der GPR ging 2019 an Herrn Dr. Christian Roth aus Leipzig. 


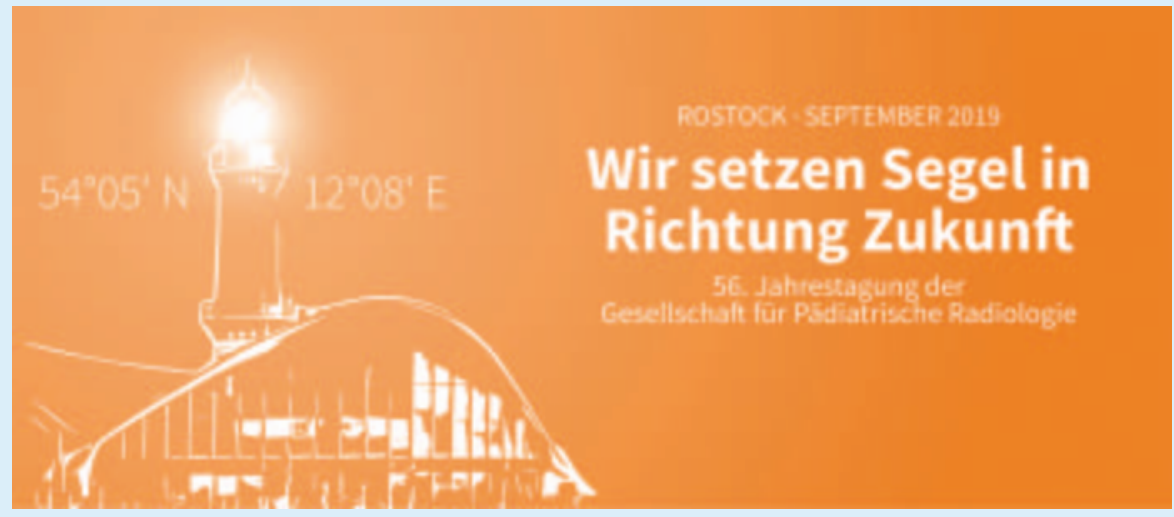

Wir danken allen Teilnehmenden, Referentinnen und Referenten sowie der Industrie für eine gelungene Veranstaltung und freuen uns Sie im kommenden Jahr in Essen wieder begrüßen zu dürfen.

Eine Fotogalerie mit Impressionen aus Rostock und Warnemünde finden Sie unter kinder-radiologie.org

Impressionen von der GPR-Jahrestagung 2019

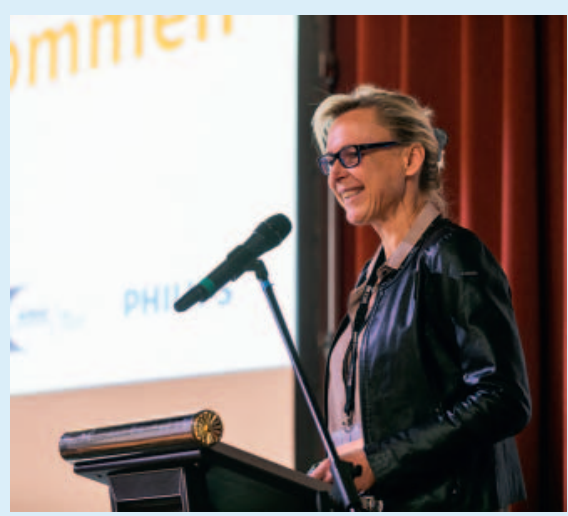

Kongresspräsidentin Dr. Christina Hauenstein bei der offiziellen Begrüßung. (c) Dirk Lässig/GPR.

(c) Dirk Lässig/GPR.

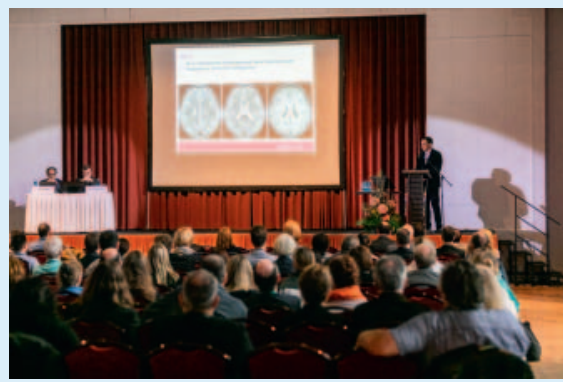

Spannende Themen und top Referenten bescherten volle Tagungsräume. (c) Dirk Lässig/GPR.

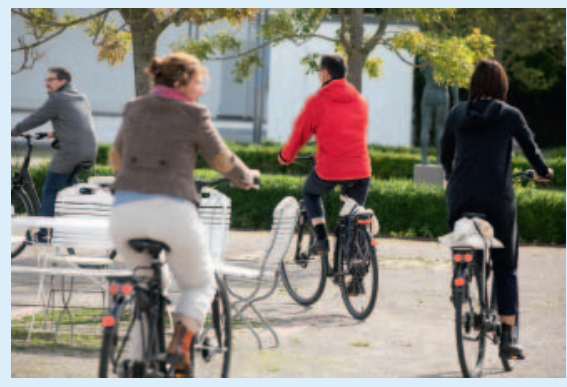

Beinahe schon eine Tradition: Die Fahrradtour mit der Kongresspräsidentin.

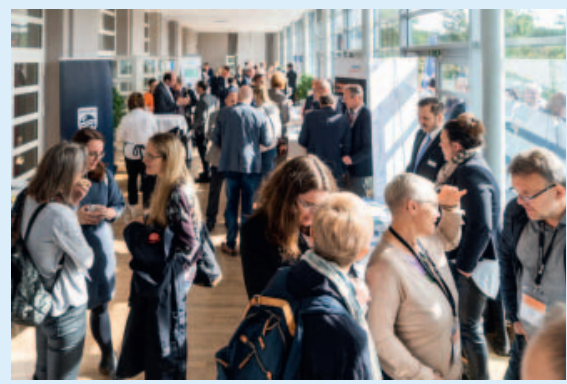

Immer viel los in den Gängen: Eine breit aufgestellte Industrieausstellung präsentierte den Besucherinnen und Besuchern die neusten Entwicklungen in Technik und Wissenschaft. @ Dirk Lässig/GPR.

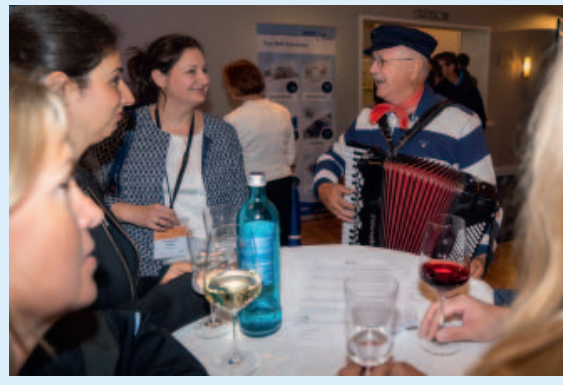

Echt nordisches Flair auf der GPR-Jahrestagung in Rostock und Warnemünde. (c) Dirk Lässig/GPR.

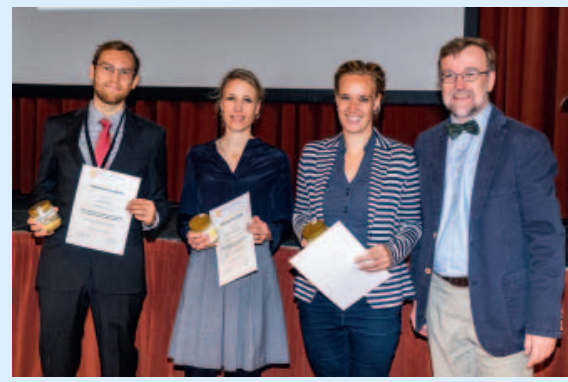

Die Preisträger des diesjährigen GPRVortragspreises (v. li n. re): Dennis Dünger, PD Dr. Wibke Uller und Dr. Hanne Kirsch mit GPR-Präsident Prof. Dr. Hans-Joachim Mentzel. @ Dirk Lässig/GPR.

SAVE THE DATE -

Jahrestagung 2020

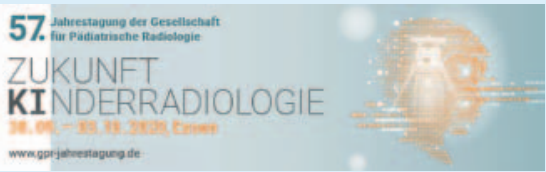

30.09. bis 3.10.2020 in Essen

Kongresspräsident:

Dr. med. Bernd Schweiger 